\title{
Implicit motor imagery performance is impaired in people with chronic, but not acute, neck pain
}

\author{
Sarah B Wallwork ${ }^{1}$, Hayley B Leake ${ }^{2}$, Aimie Peek ${ }^{3}$, G. Lorimer Moseley ${ }^{2,4}$, Tasha R Stanton ${ }^{\text {Corresp. } 2,4}$ \\ 1 University of Canberra Research Institute for Sport and Exercise, University of Canberra, Canberra, Australian Capital Territory, Australia \\ 2 IIMPACT in Health, University of South Australia, Adelaide, South Australia, Australia \\ 3 Faculty of Health Sciences, University of Sydney, Sydney, New South Wales, Australia \\ 4 Neuroscience Research Australia, Randwick, New South Wales, Australia \\ Corresponding Author: Tasha R Stanton \\ Email address: tasha.stanton@unisa.edu.au
}

Background: People with chronic neck pain have impaired proprioception (i.e., sense of neck position). It is unclear whether this impairment involves disruptions to the proprioceptive representation in the brain, peripheral factors, or both. Implicit motor imagery tasks, namely left/right judgements of body parts, assess the integrity of the proprioceptive represention. Previous studies evaluating left/right neck judgements in people with neck pain are conflicting. We conducted a large online study to comprehensively address whether people with neck pain have altered proprioceptive representation.

Methods : People with and without neck pain completed online left/right neck judgement tasks followed by a left/right hand judgement task (control). Participants judged whether the person in the image had their head rotated to their left or right side (neck task) or whether the image was of a left hand or a right hand (hand task). Participants were grouped on neck pain status (no pain; $<3$ months - acute; $\geq 3$ months - chronic) and pain location (none, left-sided, right-sided, bilateral). Outcomes included accuracy (primary) and response time (RT; secondary). Our hypotheses - that (i) chronic neck pain is associated with disrupted proprioceptive representation and (ii) the disruption is dependent on the side of usual pain, were tested with separate ANOVAs.

Results : Of 1404 participants, 105 reported acute neck pain and 161 reported chronic neck pain. When grouped on neck pain status, people with chronic neck pain were less accurate than people without neck pain $(p=0.001)$ for left/right neck judgements, but those with acute neck pain did not differ from those without neck pain $(p=0.14)$ or with chronic neck pain $(p=0.28)$. Accuracy of left/right hand judgements did not differ between groups $(p=0.58)$. RTs did not differ between groups for any comparison. When grouped on neck pain location, people were faster and more accurate at identifying right-turning neck images than left-turning neck images, regardless of history or location of pain ( $<<0.001$ for both); people with no pain were more accurate and faster than people with bilateral neck pain $(p=0.001, p=0.015)$ and were faster than those with left-sided neck pain $(p=0.021)$; people with right-sided neck pain were more accurate than people with bilateral neck pain $(p=0.018)$. Last, there was a significant interaction between neck image and side of neck pain: people with right-sided neck pain were more accurate at identifying right-sided neck turning images than people with left-sided neck pain $(p=0.008)$, but no different for leftsided neck turning images $(p=0.62)$.

Conclusions: There is evidence of impaired implicit motor imagery performance in people with chronic neck pain which may suggest disruptions to proprioceptive representation of the neck. These disruptions

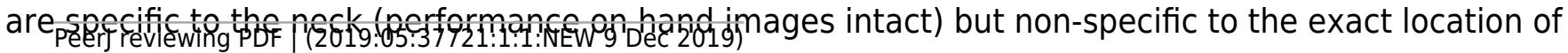


neck pain. 
1 PeerJ

2

3 Implicit motor imagery performance is impaired in people with chronic, but not acute, neck pain

5 Short title: Motor imagery performance in neck pain

${ }^{1}$ IIMPACT in Health, University of South Australia, Adelaide, South Australia, Australia

2 University of Canberra Research Institute for Sport and Exercise, University of Canberra,

13 Canberra, Australian Capital Territory, Australia

${ }^{3}$ Faculty of Health Sciences, University of Sydney, Sydney, New South Wales, Australia

${ }^{4}$ Neuroscience Research Australia, Randwick, New South Wales, Australia

17 Corresponding Author:

18 Tasha R Stanton

19 The University of South Australia

20 School of Health Sciences - CEA-14

21 GPO Box 2471, Adelaide, South Australia 5001

22 Phone: +618 83022090

23 Fax: +61883022853

24 Email: Tasha.stanton@unisa.edu.au 


\section{Abstract}

Background: People with chronic neck pain have impaired proprioception (i.e., sense of neck position). It is unclear whether this impairment involves disruptions to the proprioceptive representation in the brain, peripheral factors, or both. Implicit motor imagery tasks, namely left/right judgements of body parts, assess the integrity of the proprioceptive represention. Previous studies evaluating left/right neck judgements in people with neck pain are conflicting. We conducted a large online study to comprehensively address whether people with neck pain have altered proprioceptive representation.

Methods: People with and without neck pain completed online left/right neck judgement tasks followed by a left/right hand judgement task (control). Participants judged whether the person in the image had their head rotated to their left or right side (neck task) or whether the image was of a left hand or a right hand (hand task). Participants were grouped on neck pain status (no pain; <3 months - acute; $\geq 3$ months - chronic) and pain location (none, left-sided, right-sided, bilateral). Outcomes included accuracy (primary) and response time (RT; secondary). Our hypotheses - that (i) chronic neck pain is associated with disrupted proprioceptive representation and (ii) the disruption is dependent on the side of usual pain, were tested with separate ANOVAs.

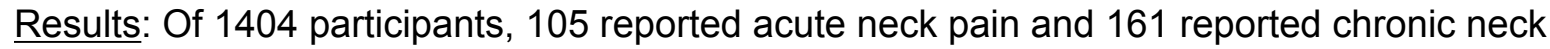
pain. When grouped on neck pain status, people with chronic neck pain were less accurate than people without neck pain $(p=0.001)$ for left/right neck judgements, but those with acute neck pain did not differ from those without neck pain $(p=0.14)$ or with chronic neck pain $(p=0.28)$. Accuracy of left/right hand judgements did not differ between groups $(p=0.58)$. RTs did not differ between groups for any comparison. When grouped on neck pain location, people were faster and more accurate at identifying right-turning neck images than left-turning neck images, regardless of history or location of pain ( $p<0.001$ for both); people with no pain were more 
50 accurate and faster than people with bilateral neck pain $(p=0.001, p=0.015)$ and were faster

51 than those with left-sided neck pain $(p=0.021)$; people with right-sided neck pain were more

52 accurate than people with bilateral neck pain $(p=0.018)$. Last, there was a significant interaction

53 between neck image and side of neck pain: people with right-sided neck pain were more

54 accurate at identifying right-sided neck turning images than people with left-sided neck pain

$55(p=0.008)$, but no different for left-sided neck turning images $(p=0.62)$.

56 Conclusions: There is evidence of impaired implicit motor imagery performance in people with

57 chronic neck pain which may suggest disruptions to proprioceptive representation related to the

58 neck. These disruptions are specific to the neck (performance on hand images intact), but non-

59 specific to the exact location of neck pain.

60 Keywords: left/right judgements, psychophysiology, Implicit motor imagery, pain, cortical body

61 representation, proprioceptive representation, working body schema 
62

63

64

\section{Introduction}

Neck pain affects $10-20 \%$ of the population in any given year (Hoy, Protani et al. 2010) and results in impaired movement and proprioception (i.e., the sense of where your body is located is space). A recent systematic review found that people with chronic neck pain have lower proprioceptive acuity than people without pain (Stanton, Leake et al. 2016). What remains unclear, however, is where the dysfunction in proprioception lies. While commonly assumed to be attributable to peripheral dysfunction (i.e., disrupted input from proprioceptors in the muscle, ligament, and skin due to injury or the presence of nociception), other possible contributions include problems with spinal processing and the encoding of proprioceptive data in the brain. In fact, people with neck pain may have intact detection and transmission of proprioceptive input, but have disruptions to the cortical proprioceptive representations that allow for planning, executing and coordinating movements (also termed the 'working body schema' (Parsons 2001)). Given that different treatments can be used to target different proprioceptive impairments, filling this research gap has both mechanistic and therapeutic importance.

Investigating whether impaired cortical proprioceptive representation underlies proprioceptive dysfunction is challenging. Functional neuroimaging can provide key information about changes in primary motor cortex representation of a movement pattern (Elgueta-Cancino, Schabrun et al. 2018), or in the cortical activation patterns (including functional connectivity) during tasks such as imagined movements (Lotze, Montoya et al. 1999). However, to date there is no method to image proprioceptive representations and thus, behavioural tasks are used. Implicit motor imagery is the most established method to interrogate the cortical proprioceptive representation and left/right judgements of pictured body parts is the most studied (Schwoebel, Coslett et al. 2002, Coslett, Medina et al. 2010, Dey, Barnsley et al. 2012, Wallwork, Butler et al. 2015) but not the only approach, for example, see (Moseley and Brugger 2009). In left/right judgments, people identify, as quickly and as accurately as possible, whether images of the target body-part 
88 (e.g., a hand) belongs to the left-side or the right-side of the body. In completing this task, the person mentally manoeuvres their own hand into the posture seen in the picture; a process supported by via neuroimaging evidence of activation of motor related areas during the task (Parsons, Fox et al. 1995, Michelon, Vettel et al. 2006). When the target body-part resides in the midline, such as for the neck and back, the task has been adapted and a judgement is made about whether the person in the image has their head or trunk rotated or laterally flexed to the left-side or right-side. Performance (accuracy) in this task is likely to be dependent on an intact neural proprioceptive representation for the target body-part, i.e., the neural representations that coordinate, plan and execute movement (Bray and Moseley 2011), although recent work has questioned whether this occurs as strongly for body parts that reside in the midline - see Alazmi, Gadsby et al. (2018). Notably, because real movement is not permitted during these task, peripheral/spinal contributors to proprioception are limited (although see Silva, Loubinoux et al. (2011) for evidence that some peripheral contribution still exists), thus the task is thought to primarily target cortical proprioceptive representations.

Previous work evaluating implicit motor imagery performance in people with neck pain has found conflicting evidence. People with recurrent neck pain $(n=30)$ were significantly less accurate than pain-free controls on a neck left/right judgement task (Elsig, Luomajoki et al. 2014), but people with chronic whiplash associated disorders $(n=64)$ were not different from controls (Pedler, Motlagh et al. 2013). However, Elsig et al (2015) evaluated performance only on a neck left/right judgement task, making it unclear whether the impaired performance is specific to the painful body part (discrete dysfunction of neck proprioceptive representation), or merely represents impaired spatial performance (regardless of the body part image used) or indeed impaired central nervous system processing. Given this uncertainty and the relatively modest sample sizes of previous work, it is key to evaluate implicit motor imagery performance 
113 in people with neck pain using a larger sample to comprehensively explore the presence and

114 nature of any dysfunction.

115

116 Here we describe a large, online study recruiting a representative sample of people with neck

117 pain and healthy pain-free controls who performed left/right judgements of neck rotation and of

118 hands. First, we extended past work by investigating the contribution of pain duration and pain

119 location on task performance, as has been investigated in people with and without back pain

120 (Bowering, Butler et al. 2014). Second, we evaluated whether impairment on the left/right

121 judgement task is location-or movement-specific. In people with pathological arm pain, impaired

122 performance on a hand left/right judgement task is specific to images that correspond to their

123 painful hand (Moseley 2004). It is unknown if a similar effect would occur in people with neck

124 pain (e.g., left sided-pain, impaired performance only on images of left neck rotation), but if

125 present, it would suggest a more nuanced dysfunction in proprioceptive representation than

126 previously realised.

127

128

We had two main aims: to determine whether neck pain and its duration are associated with impaired left/right neck rotation judgement performance (Aim 1), and to determine whether the location/side of neck pain is associated with impaired performance for left-turning and rightturning neck rotation images (Aim 2). Within Aim 1, we included an analysis with left/right hand judgements as we wanted to determine whether people with neck pain are less accurate on left/right neck rotation judgements, but not left/right hand judgements, than healthy controls. If performance was also reduced in a hand task, this would imply a more global problem. This would demonstrate that any problems with neck motor imagery are not specific to the neck and may therefore simply reflect forced-choice response time impairment (i.e., generally poor at the task). For Aim 1, we hypothesised that the presence and duration of neck pain would affect performance in a left/right neck rotation judgement task, but not a left/right hand judgement task. 
139 Specifically, we hypothesised that people with chronic neck pain would be less accurate and

140 take longer to respond to images in the left/right neck judgement task than people with no neck

141 pain or people with acute neck pain (Hypothesis 1). For Aim 2, we hypothesised that the usual

142 location of neck pain would affect performance to images showing neck rotation towards their

143 painful side (i.e., people with left-sided neck pain would be less accurate and take longer to

144 respond to images of necks turning to the left and vice versa; Hypothesis 2).

\section{Methods}

\section{Participants}

148 The current study is based on data collected as part of a large, online cross-sectional study, 149 from which characteristics of the task and normative data have been published (Wallwork, 150 Butler et al. 2013). That study collected data from a convenience sample of 1737 participants, 151 from 40 countries, who were recruited via email, using the Neuro Orthopaedic Institute (NOI, 152 Adelaide, Australia) mailing list, and via social media. Access to a computer with internet access was required to participate. Ethical approval was granted from the University of South Australia Human Research Ethics Committee (Protocol ID HS13-2009). All participants provided informed consent online, as per the Declaration of Helsinki.

\section{Questionnaires}

158 Participants completed an online questionnaire, providing details on their age, gender, and 159 handedness (Wallwork, Butler et al. 2013). This study concerns participant responses to 160 questions about neck pain. If a participant reported neck pain, they also answered questions 161 about the duration of their neck pain, the side of neck pain (left, right or bilateral), and whether 162 their neck pain was evoked by neck movement (left, right or bilateral). 
165

166

167

168

169

170

171

172

173

174

175

176

177

178

179

180

181

182

183

184

185

186

187

188

189

190

Once participants had completed the baseline questionnaire, instructions on how to complete the left/right judgement tasks using the Recognise platform (Neuro Orthopaedic Institute, Adelaide, Australia; www.noigroup.com) were provided. Two sets of images (head/neck; hands) were used for testing and the images in each set were presented in a randomised order. The first set displayed images of people's head and upper torso with their head rotated either towards their left side or their right side (i.e., left and right neck rotation, respectively). The second set displayed images of left and right hands. Participants were instructed to make a judgement on whether the person in the image was rotating their neck to their left or right side, or whether the hand was a left hand or a right hand, respectively. Participants were advised to make a left-sided response by depressing the 'a' key with their left index finger and to make a right-sided response by depressing the 'd' key with their right index finger. Participants were instructed to make a decision as quickly and as accurately as possible and to avoiding guessing. They were informed that they would have a maximum of five seconds per image to respond before the test was automatically advanced and the next image was shown.

Two trial images (either of a left and a right neck rotation or of a left and a right hand) preceded formal testing in order to orientate the participant to the keyboard commands - data were not recorded on these images. The formal test included five left/right judgement tasks, each involving 40 images. The first four left/right judgement tasks displayed images of a head and torso (left/right neck rotation judgements), the fifth task displayed images of hands (left/right hand judgements). The images were randomly presented to be left and right rotation (neck images) or sides (hand images) with images rotated at: $0^{\circ}, 90^{\circ}, 180^{\circ}$, and $270^{\circ}$. Each task comprised $50 \%$ male models and $50 \%$ female models, both aged in their early 20 's wearing plain black clothing. All left images were mirrored reflection of right images and each participant received all the images (order randomised). See Figure 1 for sample images. 
191

192

193

194

195

196

197

198

199

200

201

202

203

204

205

206

207

208

209

210

211

212

213

214

215

216

\section{INSERT FIGURE 1A-F HERE}

\section{Data processing}

Left/right judgement performance was analysed using the data from the second and fifth left/right judgement tasks. The first and third tasks were included in as methodological controls for task features and the fourth task was included to address a separate question that was unrelated to the aims of the present study. Specifically, the first left/right judgement task (neck images) was included to familiarise participants with the task and to allow for a known learning effect (Boonstra, de Vries et al. 2012). The third task (identical to task 2) was included as a backup in the situation that people with pain had difficulty completing task 2 and there were significant missing data. Because there were sufficient data available for task 2 , we decided (prior to analysis) to not include data from the third task to minimise potential participant fatigue that was likely to occur given it was the third repetition of neck images. The fourth task was a separate task that included contextual images (with various backgrounds and distractions). Data from the fifth left/right judgement task (hand images) were analysed as a control because the task involved implicit motor imagery of a remote body part, and the use of new images (hands versus necks) reduced potential for fatigue.

Participants' data were excluded if the second and fifth left/right judgement task were not completed in their entirety. Consistent with previous literature, response times were calculated only for images for which the response was correct and data from images were excluded if the response time was less than $500 \mathrm{~ms}$ as this was considered too short of a time to make a judgement response and therefore would likely represent a guess (Kunde 2001). Further, if the response time for eight consecutive images reached this 5 second limit (i.e., the participant timed-out) then the data from those images were excluded because it was assumed the participant was distracted or the internet/computer failed. We included all other responses that 
217 were 5 seconds (i.e., timed-out response) as it was assumed the participant simply took that

218 long to respond or was unsure about that particular response. Last, data sets were excluded

219 where participants did not provide necessary information for covariate analysis (age, gender,

220 handedness) or group allocation (i.e., neck pain). At the completion of data processing, there

221 were 1404 complete participant datasets.

222

223 Group allocation

224 Participants were grouped based on: (1) duration of pain (i.e., no pain; pain for less than 3

225 months considered "acute pain"; pain for 3 months or more considered "chronic pain”); (2)

226 location of pain (i.e., left-sided neck pain, right-sided neck pain, bilateral neck pain and no neck

227 pain).

228

229

Statistical analysis

230

All statistics were performed using SPSS 23.0.0 (SPSS, Chicago, IL, USA). Accuracy and

231

response time data were both tested for normality. Accuracy of responses were not normally

232

distributed and as a result were log transformed. The log transformed accuracy values met

233

normality criteria (as assessed by visual inspection of P-P plots and non-significant Shapiro-Wilk

234

statistic) and were used for all analyses. Thus, for accuracy data, the analysis results were back

235

transformed to provide group specific data (mean, $95 \% \mathrm{Cl}$ ). The back transformed mean

236

differences and their $95 \% \mathrm{Cls}$ were not reported, because the difference between logarithms of

237

two geometric means results in a logarithm of their ratio, not of their difference (Bland and

238

Altman 1996). Age, gender and handedness are known to affect left/right neck judgement

239

performance (Wallwork, Butler et al. 2013); response time increases with age, is greater in

females, and is greater in left-handed people, and accuracy reduces with age. Therefore, these

variables were considered in all analyses and included as co-variates where appropriate.

242 Further, a linear regression was performed using accuracy and response time to assess for a 
243

244

245

246

247

248

249

250

251

252

253

254

255

256

257

258

259

260

261

262

263

264

265

266

267

268

possible speed-accuracy trade-off (i.e., faster performance but incorrect response) which would suggest improper performance of the task. In all analyses, the alpha level was set at 0.05 , with a Holm-Bonferroni correction used for all multiple comparisons (Holm 1979).

To determine whether neck pain and its duration is associated with impaired left/right judgement performance (Aim 1), univariate ANOVAs were conducted (one each for accuracy and response time) for neck images, with a between-subjects main effect of Pain Duration (no pain, acute pain and chronic pain) and Age as a covariate. If a significant main effect, independent t-tests were used to make specific between group comparisons. Identical analyses were completed for left/right judgement performance on hand images (control).

To determine whether the location of neck pain is associated with impaired performance for responses to left-turning and right-turning neck images (Aim 2), accuracy and response time were separately investigated using a 2 (within-subjects main effect of Side of Head Turn in Image: left-sided turning images and right-sided turning images) by 4 (between-subjects main effect of Location of Pain: no pain, left-sided pain, right-sided pain, bilateral pain) repeated measures ANOVA. Such an analysis allowed us to determine if those with bilateral pain were equally impaired for left vs right images as well as explore whether or not there were task-based features that influenced performance, regardless of the presence or location of neck pain. Therefore, in addition, to specifically evaluate the effect of lateralised pain on performance, repeated measures ANOVAs were completed comparing only those with lateralised neck pain (left-sided neck pain versus right-sided neck pain) for performance (accuracy and response time) on left-sided turning and right-sided turning neck images.

Given that people with neck pain could have neck pain in one location (i.e., left side neck pain), but experience pain with a movement in the opposite direction (i.e., experience left-sided neck 
269 pain when rotating the neck to the right), we ran a sensitivity analysis classifying participants

270 into groups based on the direction of neck rotation which induced their pain. This was

271 completed to ensure that we did not miss a potential effect of the location of neck pain (see

272 Supplementary).

273

274 Results

275 Of the1737 people who completed the online task, 333 were excluded due to incomplete data

276 for the neck pain questionnaires. This resulted in a sample of 1404 participants from 35

277 countries. In Test 2, 546 of the 56160 single responses (i.e., $<1 \%$ of responses) were eliminated

278 and in Test 5,601 of the 56160 single responses (i.e., $\sim 1 \%$ of responses) were eliminated due

279 to too short of a response time or 8 consecutive timing-out responses. Participant demographics

280 are shown in Table 1.

281

282

Insert Table 1 here.

283

284

Accuracy-response time trade off

285

People who responded faster were also more accurate $\left(p<0.001, R^{2}=0.123\right)$. That is, there was

286

no accuracy-response time trade-off.

287

288

Aim 1: The effect of neck pain and its duration on left/right judgement performance

\section{Accuracy:}

290 Neck images: Controlling for age, there was a main effect of Pain Duration $\left(F_{2,1400}=6.36\right.$,

$291 p=0.002$, partial $\eta^{2}=0.009$ ) on accuracy of identifying direction of head turn (see Figure 2A).

292 People with no pain $(89.1 \%, 95 \% \mathrm{Cl} 88.1 \%$ to $90.1 \%)$ were more accurate $(p=0.001)$ than

293 people with chronic neck pain $(83.9 \%, 95 \% \mathrm{Cl} 81.2 \%$ to $86.7 \%)$, but were no different $(p=0.14)$

294 from people with acute neck pain $(86.2 \%, 95 \% \mathrm{Cl} 82.9 \%$ to $89.7 \%)$. Further, there was no 
295

296

297

298

299

300

301

302

303

304

305

306

307

308

309

310

311

312

313

314

315

316

317

318

319

difference in accuracy for judgements of neck images between those with acute neck pain and those with chronic neck pain $(p=0.28)$.

Hand images: Controlling for age, there was no main effect of Pain Duration $\left(F_{2,1400}=0.539\right.$, $p=0.58$, partial $\eta^{2}=0.001$ ) on accuracy for left/right judgements of hand images (see Figure $2 B$ ).

People with no pain had a mean accuracy score of $87.3 \%(95 \% \mathrm{Cl} 86.7 \%$ to $88.1 \%)$, while people with acute and chronic neck pain had a mean accuracy score of $86.3 \%(95 \% \mathrm{Cl} 84.1 \%$ to $88.7 \%)$ and $86.7 \%(95 \% \mathrm{Cl} 84.7 \%$ to $88.5 \%)$, respectively.

Insert Figure 2A-2D here

\section{Response time:}

Neck images: Controlling for age, there was no main effect of Pain Duration $\left(F_{2,1400}=1.511\right.$, $p=0.221$, partial $\eta^{2}=0.002$ ) on response time for judgements of images of necks (see Figure 2C). People with no pain were no faster at identifying an image of a neck than those with acute pain (mean difference: $-61.5 \mathrm{~ms}, 95 \% \mathrm{Cl}-156.9$ to $33.9 \mathrm{~ms}$ ) or those with chronic pain (mean difference: $-53.7 \mathrm{~ms}, 95 \% \mathrm{Cl}-133.9$ to $26.4 \mathrm{~ms}$ ) and those with acute pain were no different than those with chronic pain (mean difference: $7.7 \mathrm{~ms}, 95 \% \mathrm{Cl}-110.4$ to $125.9 \mathrm{~ms}$ ).

Hand images: Controlling for age, there was no main effect of Pain Duration ${ }_{(F 2,1400}=1.491$, $p=0.225$, partial $\eta^{2}=0.002$ ) on response time for hand images (see Figure $2 D$ ). People with no pain were no quicker at making judgements than those with acute pain (mean difference: $73.5 \mathrm{~ms}, 95 \% \mathrm{Cl}-183.6 \mathrm{~ms}$ to $36.5 \mathrm{~ms}$ ) or those with chronic pain (mean difference: $-59.1 \mathrm{~ms}$, $95 \% \mathrm{Cl}-151.5 \mathrm{~ms}$ to $33.4 \mathrm{~ms})$, and those with acute pain were no quicker than those with chronic pain (mean difference: $14.5 \mathrm{~ms}, 95 \% \mathrm{Cl}-121.9 \mathrm{~ms}$ to $150.9 \mathrm{~ms}$ ). 
Aim 2: The effect of location of neck pain on judgements to left turning and right turning neck

321 images

\section{Accuracy:}

323 When considering neck pain in all locations (none, left, right, bilateral), there was a main within-

324 subjects effect of Direction of Image Head Rotation $\left(F_{1,1345}=57.44, p<0.001\right.$, partial $\left.\eta^{2}=0.041\right)$

325 and a main between-subjects effect of Location of Pain $\left(F_{3,1345}=4.34 ; p=0.005\right.$, partial $\eta^{2}$

$326=0.010)$, but no Direction of Image Head Rotation $x$ Location of Pain interaction $\left(F_{3,1345}=2.07\right.$,

$327 p=0.103$. partial $\left.\eta^{2}=0.005\right)$. Post-hoc analyses revealed that people were less accurate at

328 identifying a left-turning neck than a right-turning neck $(p<0.001)$, that people with no pain were

329 more accurate than people with bilateral pain $(p=0.001)$, and that people with right-sided pain

330 were more accurate than people with bilateral pain $(p=0.018$; see Figure $3 A)$.

331

332

Insert Figure $3 A$ and $3 B$ here

333

334

When comparing accuracy in only those people with lateralised neck pain (i.e., only left- or right-

335

sided neck pain), there was a main effect of Direction of Image Head Rotation $\left(F_{1,150}=37.7\right.$,

336 $p<0.001$, partial $\left.\eta^{2}=0.201\right)$, but no between-subjects effect of Side of Pain $\left(F_{1,150}=2.53 ; p=\right.$

0.11 , partial $\eta^{2}=0.017$ ). There was also a significant Direction of Image Head Rotation $x$ Side of

Pain interaction $\left(F_{1,150}=7.81, p=0.006\right.$, partial $\left.\eta^{2}=0.050\right)$. To explore the interaction effect, post-

hoc tests evaluated the effect of image in each group and the effect of group for each image. In

regards to the former, post-hoc tests confirmed that both those with left-sided pain and right-

sided pain were less accurate for left-turning images than right-turning images $(p=0.018$ and $p<0.001$, respectively). In contrast, post-hoc tests exploring the effect of group for each image show that accuracy was no different between groups for left-turning neck images $\left(t_{1,150}=-0.504\right.$, $p=0.62)$, whereas for right-turning neck images those with right-sided neck pain were significantly more accurate $\left(t_{1,150}=-2.70, p=0.008 ; 93.1 \%, 95 \% \mathrm{Cl} 89.9 \%\right.$ to $\left.96.2 \%\right)$ than those 
346 with left-sided neck pain $(87.3 \%, 95 \% \mathrm{Cl} 84.5 \%$ to $90.2 \%$; see Figure $4 \mathrm{~A})$. Overall, this suggests

347 that people with right-sided neck pain have a larger difference in accuracy between right- and

348 left-turning images than do people with left-sided neck pain, and this larger difference is driven

349 by improved performance for right-turning neck images.

350

351 Insert Figure $4 A$ and $4 B$ here

352

353 Response time:

354 Similar to accuracy findings, there was a main within-subjects effect of Direction of Image Head

355 Rotation $\left(F_{1,1347}=18.60, p<0.001\right.$, partial $\left.\eta^{2}=0.014\right)$ and a main between-subjects effect of

356 Location of Pain $\left(F_{3,1347}=3.51, p=0.015\right.$, partial $\left.\eta^{2}=0.008\right)$, but no Direction of Image Head

357 Rotation $x$ Location of Pain interaction $\left(F_{3,1347}=1.56, p=0.20\right.$, partial $\left.\eta^{2}=0.003\right)$. Post-hoc

358 analyses revealed that people were faster at identifying a right-turning neck image than a left-

359 turning neck image (mean difference $=-52.02 \mathrm{~ms}, 95 \% \mathrm{Cl}=-75.68 \mathrm{~ms}$ to $-28.36 \mathrm{~ms}, \mathrm{p}<0.001$ ), and

360 that people with no pain were faster at responding to images than people with left-sided neck

361 pain (mean difference $=-32.6 \mathrm{~ms}, 95 \% \mathrm{Cl}-245.4 \mathrm{~ms}$ to $-19.8 \mathrm{~ms}, \mathrm{p}=0.021$ ) and people with

362 bilateral neck pain (mean difference $=-231.4 \mathrm{~ms}, 95 \% \mathrm{Cl}-231.3 \mathrm{~ms}$ to $25.2 \mathrm{~ms}, \mathrm{p}=0.015$; see

363 Figure 3B). When only those with lateralised neck pain (left- or right-sided pain) were included in

364 the analysis, findings were unchanged, although the interaction between Direction of Image

365 Head Rotation $x$ Side of Pain approached significance $\left(F_{1,150}=3.66, p=0.058\right.$, partial $\eta^{2}=0.024$;

366 see Figure 4B and Supplemental File for full analysis).

367

368 Sensitivity analyses: The results of analyses evaluating the left/right judgement performance

369 effects of neck pain evoked by side-specific movement (e.g., pain evoked with head/neck

370 movement to the left, head/neck movement to the right, both, or neither) were consistent with

371 the typical location of neck pain results reported above. See Supplementary File for full analysis 
372 results. Supplementary Figures 1A (accuracy), 1B (response time) show results considering full

373 sample (no pain with neck rotation, neck pain with left head rotation, neck pain with right head

374 rotation, neck pain with head rotation in both directions) and Supplementary Figures 2A

375 (accuracy), 2B (response time) show results limited to those with neck pain induced by right or 376 left head rotation.

Interim summary: Taken together, people were faster and more accurate in identifying a rightturning neck image than a left-turning neck image, regardless of whether or not they experienced neck pain. People with bilateral pain were most impaired - they were less accurate and slower at making neck left/right judgements than those with no pain (and less accurate than those with right-sided pain). People with left-sided pain were slower at identifying neck images than those with no pain, but no less accurate. Last, people with unilateral pain had varying accuracy based on the location of neck pain-side: those with right-sided neck pain were more accurate at identifying right-turning neck images than those with left-sided neck pain (but did not differ for left-turning neck images nor for any comparison of reaction time).

\section{Discussion}

We hypothesised that people with chronic neck pain would have impaired performance on a left/right neck rotation judgement task (versus those without neck pain and those with acute neck pain), but not on a left/right hand judgement task (Aim 1). Our hypothesis was partially supported. As hypothesised, people with chronic neck pain were less accurate than people without neck pain on a left/right neck rotation judgement task, but not on a left/right hand judgement task. However, people with chronic neck pain were no less accurate than those with acute neck pain and response time was unaffected by the presence or duration of neck pain. Our second hypothesis, that the location of neck pain-would impair performance on images showing neck rotation towards the painful side (Aim 2), was not supported. We found only group 
398

399

400

401

402

403

404

405

406

407

408

409

410

411

412

413

414

415

416

417

418

419

420

421

422

423

differences based on pain location (those with bilateral pain were less accurate and slower than 'no pain' controls, left-sided pain were slower than 'no pain' controls). A significant interaction between side of pain and image (left/right neck rotation) found the opposite of what we predicted: people with right-sided neck pain were more accurate at identifying an image of a right-turning head than those with left-sided neck pain.

Taken together, the current findings support the possibility of impaired neural proprioceptive representation of the neck in people with chronic neck pain, but also show that overall, the impairment is not specific to the painful side of the neck. The distribution or spread of pain may be important, given that those with bilateral pain had consistently worse performance. These results have important theoretical and clinical implications.

\section{Effect of Pain Duration on Task Performance:}

There is accumulating evidence that people with chronic pain are less accurate at left/right judgement tasks that use images that correspond to the painful body-part (Breckenridge, Ginn et al. 2018), indicating disruptions to neural proprioceptive representations that are associated with movement. Our findings are consistent with this body of literature and specifically support previous work in people with recurrent, idiopathic neck pain that showed lower accuracy at a left/right neck judgement task, than that observed in people without neck pain (Elsig, Luomajoki et al. 2014). That similar impairments in accuracy were not detected in one small study in people with chronic whiplash associated disorder (WAD) (Pedler, Motlagh et al. 2013), might suggest differences exist within people with neck pain, between those with and without WAD. However, there are two issues that suggest otherwise. First, the study by Pedler et al. (2013) involved a different task - judging whether an image showed the left or right side of the neck, in contrast to our task - judging whether an image showed the neck and head rotated to the left or the right. It seems probable that the tasks interrogate different processes. Further, it was not 
424 powered to detect a difference (only 24 participants in the control group), but instead powered to

425 detect a correlation between performance in left/right judgments and factors that are thought to

426 be predictive of non-recovery (Pedler, Motlagh et al. 2013). Second, it is highly likely that our

427 sample included a large number of people with WAD, but our online design meant we could not

428 assess them, nor definitively classify people as having WAD or not.

Reduced accuracy in the neck left/right judgement task, but not in the hand task, supports the somatotopically specific nature of impairments in proprioceptive representation. This specificity of impairment is largely consistent with past research in several chronic pain conditions,

433 including back pain (Bowering, Butler et al. 2014) and arm pain (Schwoebel, Friedman et al.

434 2001, Moseley 2004) (see also systematic review findings: (Breckenridge, Ginn et al. 2018)), 435 and also in experimentally induced pain (Moseley, Sim et al. 2005) and even the expectation of experimentally induced pain (Hudson, McCormick et al. 2006). It is important to consider the wider body of evidence when interpreting the current results because different problems might underpin impairment of accuracy as distinct from response time. Full review is beyond the scope of this paper, but deficits in accuracy are more consistent with disrupted proprioceptive representation and deficits in RT are more consistent with unequal weighting between representations of either hand, or movement (see (Moseley, Butler et al. 2012, Wallwork, Bellan et al. 2016, Wallwork, Bellan et al. 2017) for extensive reviews, although see Pelletier, Bourbonnais et al. (2018) for other possible contributors to performance).

Recent findings by Pelletier and colleagues have shown that left/right judgement performance is related to sensorimotor and cognitive function in people with pain (Pelletier, Bourbonnais et al.

447 2018). That is, performance on tasks including tactile acuity and motor function were related to 448 left/right judgement accuracy of the affected body part, as was taking pain medication.

449 Additionally, poor performance in a cognitive stroop task was associated with general impaired 
450 left/right judgement performance. Thus, it is possible that the differences observed between the

451 chronic pain and no pain groups in our study (Aim 1) could, in part, be attributable to other

452 factors (such as those reported by Pelletier and colleagues) than to impairments to the

453 proprioceptive neural representation. However, these relationships between sensorimotor

454 function and left/right judgement performance are not straightforward: in people with chronic

455 painful knee osteoarthritis, impairments in tactile acuity were not related to left/right judgement

456 performance (Stanton, Lin et al. 2013). Also, if impaired cognitive function in the chronic neck

457 pain group influenced their task performance (as per Pelletier et al), we would expect impaired

458 performance on both hand and neck image left right judgement tasks, which we did not see.

459 Further work is clearly required to understand the nuanced and complex mechanisms

460 contributing to performance in this task.

461

462 That people with neck pain have impaired accuracy on left/right judgements for neck images

463 has particular clinical relevance, because it raises the possibility that treatments aiming to

464 reverse that deficit might help to reduce pain in those with chronic neck pain. Certainly, in other

465 pain populations (i.e., complex regional pain syndrome and phantom limb pain), motor imagery

466 training, which includes left/right body part judgements, has been shown to be effective at

467 reducing pain (Moseley 2004, Moseley 2006). There are three important caveats here that urge

468 caution before adding motor imagery training to a clinical toolbox. First, it is not known whether

469 an accuracy deficit of $\sim 6 \%$ is clinically meaningful. Second, whether deficits vary according to

470 diagnosis or mechanism of injury remains to be determined. Third, any recommendations for

471 new treatments should only be made once the treatment is known to be safe and effective, as

472 determined in clinical trials.

473

474 That we found no difference in accuracy between the acute pain group and the healthy control

475 group (without neck pain) supports the idea that these impaiments do not occur in the acute 
476 stages. Such findings are largely consistent with previous work in a back pain sample showing

477 that impairments are minimal in people with current back pain (but no history of back pain)

478 (Bowering, Butler et al. 2014). However, we also found that the acute pain group did not differ in

479 accuracy to the chronic pain group either which raises the possibility that small changes in

480 performance may occur in the acute phase, but they are not large enough to be detected even

481 in a large cohort such as this, which implies any difference would be very small and likely to be

482 unimportant. Investigating longitudinal change in proprioceptive representation as pain persists

483 appears warranted because perhaps early deficit is a risk factor for poor recovery.

485 While left/right judgement accuracy was impaired, response time was not. This may be

486 explained by the different processes underpinning the two outcomes. That is, RT deficits in limb

487 pain are currently interpreted as reflecting unequal weighting of space-based or side-based

488 representations (see above). There is increasing evidence showing space-based deficits in

489 processing in association with limb pain, with a range of effects including thermoregulation,

490 motor control and tactile processing (Moseley, Gallace et al. 2009, Stanton, Lin et al. 2012,

491 Reid, Wallwork et al. 2016, Reid, Braithwaite et al. 2018)(see Moseley, Gallace et al. (2012) for

492 a review). However, in limb pain, the spatial representations seem to involve the area of

493 peripersonal space in which the limb is used. This clearly is not applicable to spinal pain, so it is 494 perhaps unsurprising that we did not observe RT deficits here.

495

496

Effect of Location/Side Specific Pain on Performance

497 Our finding that location specific neck pain (Aim 2) or movement-evoked neck pain (see

498 Supplementary material) did not affect performance to images showing neck rotation to their

499 affected side would not be predicted on the presumed relationship between proprioceptive

500 representation and motor imagery performance (Bray and Moseley 2011, Schmid and

501 Coppieters 2012, Wallwork, Bellan et al. 2016). That is, we predicted that performance on a task 
502 that requires access to the representations for a body part or movement that is normally

503 associated as being painful would be worse than for a task that requires access to the

504 representations for a body part or movement that is not normally associated as being painful.

505 Such a hypothesis is supported by past work in people with painful osteoarthritis (Stanton, Lin et

506 al. 2012) and also by contemporary theories (i.e., the cortical body matrix theory (Moseley,

507 Gallace et al. 2012)) in this area.

508

509 Contemporary theories of motor processing emphasise the distributed nature of processing and

510 the conceptual construct of neural networks (or 'neurotags' (Butler and Moseley 2003, Moseley

511 and Butler 2015)) that are under a potentially infinite number of other neural networks (see

512 Wallwork, Bellan et al. (2016) for a review). For example, a movement of neck rotation (the

513 'output'), depends on incoming data (including proprioceptive cues, visual cues [head rotation],

514 tactile cues [from skin stretching]), stored data (past experience) and predicted outcomes of a

515 movement. As part of this complex process, motor efferent copies (i.e., a copy of the motor

516 command) are created with multi-sensory feedback from the movement used to determine if the

517 movement occurred as planned. The more often the network is activated, the stronger and more

518 precise it becomes (Pearson, Finkel et al. 1987, Pilz, Veit et al. 2004), which in turn facilitates

519 activation of the 'learned' network. It might be predicted then, that when performing a left/right

520 neck rotation judgement task that uses these disrupted proprioceptive representations,

521 performance would be specifically hindered for an image congruent with painful movement -

522 even when the full movement output has not occurred (i.e., they have not actually moved). That

523 we did not consistently find an interaction effect in this study indicates that perhaps these

524 proprioceptive representations have general alterations for the affected body part, and are not

525 specific to the location of pain, or movement-evoked side of pain. Alternatively, it may be that

526 the precision with which neck proprioceptive input is represented for side-specific movements is

527 less than we would expect in lateralised body parts (e.g., left and right hand).

Peer] reviewing PDF | (2019:05:37721:1:1:NEW 9 Dec 2019) 
530 We found that people were generally more accurate at identifying images of right neck rotation

531 than left neck rotation, regardless of neck pain status. Previous studies have identified side-

532 specific effects as a function of hand dominance: right-handers are more accurate at identifying

533 right hand images than left-handers (Braithwaite et al. In Preparation)(Wallwork, Butler et al.

534 2013) and perhaps these findings transfer to our results too. In our sample, 1192 of 1404 were

535 right-handed so there was a much stronger right-handed presence in our sample group which

536 would be consistent with this idea. However, it seems unlikely that the same effect of

537 dominance extends to the neck like it does the hand. Of relevance, people are faster and more

538 accurate at a left/right judgement task when images are rotated $90^{\circ}$ clockwise (medial) than

539 when they are rotated $90^{\circ}$ anti-clockwise (lateral) for images of the neck (Wallwork, Butler et al.

540 2013) and for images of the back ( $n=1189$ participants) (Bowering, Butler et al. 2014). Given

541 that past work has also shown that such differences based on image orientation may reflect a

542 switch from implicit motor imagery to more proprioceptive-visual matching strategies (as seen in

543 hand left/right judgements) (De Simone, Tomasino et al. 2013), these hypotheses clearly

544 require further investigation.

545

546 An interaction between side of the body and side of pain was seen-only for analyses considering

547 the location of neck pain (left versus right-sided), but it was opposite to the direction

548 hypothesised. People with right-sided neck pain were more accurate at identifying an image of a

549 right-turning head than people with left-sided neck pain. While previous work by Stanton, Lin et

550 al. (2012) found that people with right-sided knee osteoarthritic pain or upper limb pain were

551 generally more accurate than those with left-sided pain at identifying images of hands or feet,

552 enhanced performance for right-sided pain $\mathrm{x}$ right sided images was not seen. Specifically,

553 those with right-sided knee or upper limb pain were no more accurate at identifying a right 
554 hand/foot than a left hand/foot, where as in participants with left-sided pain, performance was 555 most impaired for left hand or foot images versus right images (side of pain matched side of 556 impairment) (Stanton, Lin et al. 2012). Together with generalised reduction in performance for 557 left versus right-sided neck images seen here, such findings raise the possibility that there might 558 be a lateralised performance effect whereby spatial lateralisation of processing during the task 559 (i.e., in the right posterior parietal cortex) results in an interference effect with left-sided (right 560 processed) proprioceptive representations (i.e, left images), which can be further affected by 561 left-sided (right processed) pain, but is relatively spared by right-sided (left processed) pain.

562 However, why performance for right images in people with right-sided neck pain is seemingly 563 enhanced remains unclear. To our knowledge, this line of enquiry remains to be tested.

Last, we found that people with bilateral neck pain (typical and movement-evoked) were slower and less accurate than people with no neck pain. Such findings raise the possibility that the disruptions to proprioceptive representation are greater when pain is more widespread in area (i.e., bilateral). Finally, people with right-sided neck pain were more accurate than people with bilateral neck pain, but no faster than people with left-sided pain, and people with no pain were also faster than people with left-sided pain. This pattern of findings requires validation, but generally supports the largest impairments seen in those with bilateral pain, followed by leftsided pain, and the least impairment in those with right-sided pain.

\section{Study limitations}

575 While this is one of the largest left/right judgement studies undertaken to date, interpretation of 576 our results should consider several limitations. First, the online nature of the study means that 577 computer malfunctions, screen size, screen resolution, and refresh rate could increase variance 578 and reduce our power, although it is unlikely to systematically bias the results. Furthermore, 
579 being an online study, we cannot exclude that participants took part more than once or provided 580 inaccurate information when answering the online questionnaire. To combat limitations imposed 581 by this increase in variability of the data, we purposefully aimed to collect a large sample. There 582 is also the possibility that participants' responses to the fifth block (hand images) may have 583 been affected by fatigue when compared to responses to the second block (neck images).

584 Although possible, we think this is unlikely as fatigue is primarily seen when an identical task is 585 repeated. Because the hand task in the fifth block was new, we anticipate that fatigue is less of 586 a concern. If fatigue were a factor, we would expect that people with neck pain would perform 587 worse at the hand task than pain-free people (Dailey, Keffala et al. 2015), which was not 588 observed. Additionally, we were unable to explore image orientation specific performance 589 results for neck and hand images; these data were not retrievable. Given that evaluating image 590 orientation can help to interpret what might underlie accuracy impairments (e.g., impairments in

591 motor imagery or alterations in visual processing (Edwards, Causby et al. 2019)), future research that purposefully explores the influence of neck image orientation on performance is clearly needed.

Given an online study, there are also inherent limitations in participant self-report for 'acute' and 'chronic' neck pain, because people might have different interpretations. For example, despite providing clear descriptors about duration, it is possible that someone with an ongoing chronic problem might report 'acute' pain because they are suffering a recent flare-up. Such challenges may also be a potential factor in the lack of performance difference seen between acute and chronic neck pain. Furthermore, while it would have been of interest to consider pain intensity as a covariate in the analyses, limitations in the online questionnaire meant that pain intensity values were not collected for those who reported having acute or chronic pain but who only reported pain with neck movement. That is, pain intensity scores only captured current neck 603 pain. Unfortunately then, including pain as a covariate would have excluded numerous 
604 participants from the analysis. Additionally, given that past studies have shown an inconsistent 605 relationship between pain intensity and left/right judgement performance (Bray and Moseley 606 2011, Linder, Michaelson et al. 2016, Pelletier, Bourbonnais et al. 2018, Stanton, Gilpin et al. 607 2018), this makes the relevance of including pain intensity as a covariate less certain. For these 608 reasons pain intensity was not included covariate in our analyses. Last, as reported in a 609 previous study using this data (Wallwork, Butler et al. 2013), using the 'a' and 'd' keys on the 610 keyboard as a response apparatus has limitations in that both keys are located on the left of the 611 keyboard (i.e.,in the left side of space). We now understand that a bias in the allocation of 612 attention to a spatially-defined location could influence responding (Moseley, Gallace et al. 613 2012, Reid, Wallwork et al. 2016); however such influences are less likely to impact our main

614 findings, given that the neck is not like the limbs which are normally situated in one spatial field 615 (right or left of body midline) Regardless, future work in this area should aim to use keys that 616 result in the response hands being positioned on either side of the body midline. Finally, that we 617 did not lodge and lock our experimental protocol prior to conducting this experiment reflects that 618 we began the study before we fully understood the importance of doing so. This practice 619 enhances the transparency of research and is now recommended for observational and clinical 620 designs in many fields including pain (Lee, E Lamb et al. 2018).

622 Conclusions

623 In our large online cohort $(n=1404)$, including 266 people with neck pain, we found evidence of 624 disruptions to proprioceptive representations that were specific to the neck in people with 625 chronic neck pain. In particular, people with chronic neck pain were less accurate at a left/right 626 neck rotation judgement task, but not a left/right hand judgement task, than people with no neck 627 pain. Contrary to our expectations, we found that the location of neck pain-generally did not 628 influence responses to images associated with movement towards their painful side. Overall 
629 these findings indicate that investigation of a graded motor imagery program to reduce pain in

630 people with neck pain may be warranted. However, further investigations in neck pain

631 subgroups, such as whiplash associated disorder, need to be addressed.

632

633 Conflicts of interest:

634 In the last 5 years, G. Lorimer Moseley has received support from: ConnectHealth UK, Seqirus, 635 Kaiser Permanente, Workers' Compensation Boards in Australia, Europe and North America, 636 AIA Australia, the International Olympic Committee, Port Adelaide Football Club and Arsenal

637 Football Club. Professional and scientific bodies have reimbursed him for travel costs related to 638 presentation of research on pain at scientific conferences/symposia. He has received speaker 639 fees for lectures on pain and rehabilitation. He receives book royalties from NOlgroup 640 publications, Dancing Giraffe Press \& OPTP. G. Lorimer Moseley is an Academic Editor for 641 PeerJ. TRS has received support from Eli Lilly Ltd for travel and accommodation costs. 
644 Alazmi, L., G. E. Gadsby, N. R. Heneghan and T. D. Punt (2018). "Do trunk-based left/right judgment tasks 645 elicit motor imagery?" Musculoskelet Sci Pract 35: 55-60.

646 Bland, J. M. and D. G. Altman (1996). "Statistics Notes: The use of transformation when comparing two 647 means." BMJ 312(7039): 1153.

648 Boonstra, A. M., S. J. de Vries, E. Veenstra, M. Tepper, W. Feenstra and E. Otten (2012). "Using the Hand 649 Laterality Judgement Task to assess motor imagery: a study of practice effects in repeated 650 measurements." Int J Rehabil Res 35(3): 278-280.

651 Bowering, K. J., D. S. Butler, I. J. Fulton and G. L. Moseley (2014). "Motor imagery in people with a 652 history of back pain, current pain, both, or neither." The Clinical Journal of Pain 30(12): 1070-1075.

653 Bray, H. and G. L. Moseley (2011). "Disrupted working body schema of the trunk in people with back 654 pain." British Journal of Sports Medicine 45(3): 168-173.

655 Breckenridge, J. D., K. A. Ginn, S. B. Wallwork and J. H. McAuley (2018). "Do people with chronic 656 musculoskeletal pain have impaired motor imagery? A meta-analytical systematic review of the 657 left/right judgement task." The Journal of Pain.

658 Butler, D. and G. L. Moseley (2003). Explain pain. Adelaide, NOI Group Publishing.

659 Coslett, H. B., J. Medina, D. Kliot and A. Burkey (2010). "Mental motor imagery and chronic pain: The 660 foot laterality task." Journal of the International Neuropsychological Society: 1-10.

661 Dailey, D. L., V. J. Keffala and K. A. Sluka (2015). "Do cognitive and physical fatigue tasks enhance pain, 662 cognitive fatigue, and physical fatigue in people with fibromyalgia?" Arthritis care \& research 67(2): 288663296.

664 De Simone, L., B. Tomasino, N. Marusic, R. Eleopra and R. I. Rumiati (2013). "The effects of healthy aging on mental imagery as revealed by egocentric and allocentric mental spatial transformations." Acta Psychol (Amst) 143(1): 146-156.

667 Dey, A., N. Barnsley, R. Mohan, M. McCormick, J. H. McAuley and G. L. Moseley (2012). "Are children 668 who play a sport or a musical instrument better at motor imagery than children who do not?" British 669 Journal of Sports Medicine.

670 Edwards, L. M., R. S. Causby, H. Stewart and T. R. Stanton (2019). "Differential influence of habitual 671 third-person vision of a body part on mental rotation of images of hands and feet." Experimental Brain 672 Research.

673 Elgueta-Cancino, E., S. Schabrun and P. Hodges (2018). "Is the Organization of the Primary Motor Cortex 674 in Low Back Pain Related to Pain, Movement, and/or Sensation?" Clin J Pain 34(3): 207-216.

675 Elsig, S., H. Luomajoki, M. Sattelmayer, J. Taeymans, A. Tal-Akabi and R. Hilfiker (2014). "Sensorimotor 676 tests, such as movement control and laterality judgment accuracy, in persons with recurrent neck pain 677 and controls. A case-control study." Manual Therapy 19(16): 555-561.

678 Holm, S. (1979). "A Simple Sequentially Rejective Multiple Test Procedure." Scandinavian Journal of 679 Statistics 6(2): 65-70.

680 Hoy, D. G., M. Protani, R. De and R. Buchbinder (2010). "The epidemiology of neck pain." Best Pract Res 681 Clin Rheumatol 24(6): 783-792.

682 Hudson, M. L., K. McCormick, N. Zalucki and G. L. Moseley (2006). "Expectation of pain replicates the 683 effect of pain in a hand laterality recognition task: Bias in information processing toward the painful 684 side?" European Journal of Pain 10(3): 219-224.

685 Kunde, W. (2001). "Response-Effect Compatibility in Manual Choice Reaction Tasks." Journal of 686 Experimental Psychology: Human Perception and Performance 27(2): 287-394.

687 Lee, H., S. E Lamb, M. Bagg, E. Toomey, A. Cashin and L. Moseley (2018). Reproducible and Replicable 688 Pain Research: A Critical Review. 
689

690

691

692

693

694

695

696

697

698

699

700

701

702

703

704

705

706

707

708

709

710

711

712

713

714

715

716

717

718

719

720

721

722

723

724

725

726

727

728

729

730

731

732

733

734

735

Linder, M., P. Michaelson and U. Roijezon (2016). "Laterality judgments in people with low back pain--A cross-sectional observational and test-retest reliability study." Man Ther 21: 128-133.

Lotze, M., P. Montoya, M. Erb, E. Hulsmann, H. Flor, U. Klose, N. Birbaumer and W. Grodd (1999).

"Activation of cortical and cerebellar motor areas during executed and imagined hand movements: an fMRI study." J Cogn Neurosci 11(5): 491-501.

Michelon, P., J. M. Vettel and J. M. Zacks (2006). "Lateral somatotopic organization during imagined and prepared movements." J Neurophysiol 95(2): 811-822.

Moseley, G. and P. Brugger (2009). "Interdependence of movement and anatomy persists when amputees learn a physiologically impossible movement of their phantom limb." Proceedings of the National Academy of Sciences of the United States of America 106(44): 18798-18802. Moseley, G., D. Butler, T. Beames and T. Giles (2012). The graded motor imagery handbook. Adelaide, NOIgroup publishing.

Moseley, G., A. Gallace and G. D. Iannetti (2012). "Neglect-like tactile dysfunction in chronic back pain." Neurology 79: 327-332.

Moseley, G., A. Gallace and C. Spence (2009). "Space-based, but not arm-based, shift in tactile processing in complex regional pain syndrome and its relationship to cooling of the affected limb." BRAIN 132: 3142-3151.

Moseley, G. L. (2004). "Graded motor imagery is effective for long-standing complex regional pain syndrome: a randomised controlled trial." Pain 108: 192-198.

Moseley, G. L. (2004). "Why do people with complex regional pain syndrome take longer to recognize their affected hand?" Neurology 62(12): 2182-2186.

Moseley, G. L. (2006). "Graded motor imagery for pathologic pain: A randomized controlled trial." Neurology 67(12): 2129-2134.

Moseley, G. L. and D. S. Butler (2015). "15 years of Explainig Pain - the past, present and future." The Journal of Pain In Press.

Moseley, G. L., A. Gallace and C. Spence (2012). "Bodily illusions in health and disease: Physiological and clinical perspectives and the concept of a cortical 'body matrix'." Neuroscience and biobehavioral reviews 36: 34-46.

Moseley, G. L., D. F. Sim, M. L. Henry and T. Souvlis (2005). "Experimental hand pain delays recognition of the contralateral hand--Evidence that acute and chronic pain have opposite effects on information processing?" Cognitive Brain Research 25(1): 188-194.

Parsons, L. M. (2001). "Integrating cognitive psychology, neurology and neuroimaging." Acta Psychologica 107(1-3): 155-181.

Parsons, L. M., P. T. Fox, J. H. Downs, T. Glass, T. B. Hirsch, C. C. Martin, P. A. Jerabek and J. L. Lancaster (1995). "Use of implicit motor imagery for visual shape discrimination as revealed by PET." Nature 375: 54.

Pearson, J. C., L. H. Finkel and G. M. Edelman (1987). "Plasticity in the organization of adult cerebral cortical maps: a computer simulation based on neuronal group selection." J Neurosci 7(12): 4209-4223. Pedler, A., H. Motlagh and M. Sterling (2013). "Laterality judgments are not impaired in patients with chronic whiplash associated disorders." Man Ther 18(1): 72-76.

Pelletier, R., D. Bourbonnais, J. Higgins, M. Mireault, M. A. Danino and P. G. Harris (2018). "Left Right Judgement Task and Sensory, Motor, and Cognitive Assessment in Participants with Wrist/Hand Pain." Rehabilitation research and practice 2018: 1530245-1530245.

Pilz, K., R. Veit, C. Braun and B. Godde (2004). "Effects of co-activation on cortical organization and discrimination performance." NeuroReport 15(17): 2669-2672.

Reid, E., S. B. Wallwork, D. Harvie, K. J. Chalmers, A. Gallace, C. Spence and G. L. Moseley (2016). "A New Kind of Spatial Inattention Associated With Chronic Limb Pain?" Ann Neurol 79(4): 701-704. 
736
Reid, E. J., F. A. Braithwaite, S. B. Wallwork, D. Harvie, K. J. Chalmers, C. Spence, A. Gallace and G. L. Moseley (2018). "Spatially-defined motor deficits in people with unilateral complex regional pain syndrome." Cortex 104: 154-162.

Schmid, A. B. and M. W. Coppieters (2012). "Left/right judgment of body parts is selectively impaired in patients with unilateral carpal tunnel syndrome." Clinical Journal of Pain 28(7): 615-622.

Schwoebel, J., H. B. Coslett, J. Bradt, R. Friedman and C. Dileo (2002). "Pain and the body schema: Effects of pain severity on mental representations of movement." Neurology 59(5).

Schwoebel, J., R. Friedman, N. Duda and H. B. Coslett (2001). "Pain and the body schema: Evidence for peripheral effects on mental representations of movement." Brain 124(10): 2098-2104.

Silva, S., I. Loubinoux, M. Olivier, B. Bataille, O. Fourcade, K. Samii, M. Jeannerod and J. F. Demonet (2011). "Impaired visual hand recognition in preoperative patients during brachial plexus anesthesia: importance of peripheral neural input for mental representation of the hand." Anesthesiology 114(1): 126-134.

Stanton, T., C. Lin, R. Smeets, D. Taylor, R. Law and G. Moseley (2012). "Spatially-defined disruption of motor imagery performance in people with osteoarthritis." Rheumatology 51: 1455-1464.

Stanton, T. R., H. R. Gilpin, L. Edwards, G. L. Moseley and R. Newport (2018). "Illusory resizing of the painful knee is analgesic in symptomatic knee osteoarthritis." PeerJ 6: e5206-e5206.

Stanton, T. R., H. B. Leake, K. J. Chalmers and G. L. Moseley (2016). "Evidence of Impaired Proprioception in Chronic, Idiopathic Neck Pain: Systematic Review and Meta-Analysis." Physical therapy 96(6): 876887.

Stanton, T. R., C. W. Lin, H. Bray, R. J. Smeets, D. Taylor, R. Y. Law and G. L. Moseley (2013). "Tactile acuity is disrupted in osteoarthritis but is unrelated to disruptions in motor imagery performance." Rheumatology 52(8): 1509-1519.

Wallwork, S. B., V. Bellan, M. J. Catley and G. L. Moseley (2016). "Neural representations and the cortical body matrix: implications for sports medicine and future directions." Br J Sports Med 50(16): 990-996. Wallwork, S. B., V. Bellan and G. L. Moseley (2017). "Applying Current Concepts in Pain-Related Brain Science to Dance Rehabilitation." J Dance Med Sci 21(1): 13-23.

Wallwork, S. B., D. S. Butler, I. Fulton, H. Stewart, I. Darmawan and G. L. Moseley (2013). "Left/right neck rotation judgments are affected by age, gender, handedness and image rotation." Man Ther 18(3): 225230.

Wallwork, S. B., D. S. Butler, D. J. Wilson and G. L. Moseley (2015). "Are people who do yoga any better at a motor imagery task than those who do not?" Br J Sports Med 49(2): 123-127. 
Table 1 (on next page)

Demographic information of included participants

All numbers represent count data unless otherwise specified. 


\begin{tabular}{|c|c|c|c|c|c|c|c|c|c|c|c|c|}
\hline \multirow[b]{2}{*}{$\begin{array}{l}\text { Demographic } \\
\text { variables }\end{array}$} & \multirow[b]{2}{*}{$\begin{array}{c}\text { All } \\
\text { participants }\end{array}$} & \multicolumn{3}{|c|}{ Duration of pain } & \multicolumn{4}{|c|}{ Location of pain } & \multicolumn{4}{|c|}{ Movement-evoked neck pain } \\
\hline & & $\begin{array}{l}\text { No } \\
\text { pain }\end{array}$ & $\begin{array}{l}\text { Acute } \\
\text { neck } \\
\text { pain }\end{array}$ & $\begin{array}{c}\text { Chronic } \\
\text { Neck } \\
\text { pain }\end{array}$ & $\begin{array}{l}\text { No } \\
\text { pain }\end{array}$ & Left & Right & Bilateral & $\begin{array}{l}\text { No } \\
\text { pain }\end{array}$ & Left & Right & Bilateral \\
\hline TOTAL & 1404 & 1138 & 105 & 161 & 1104 & 79 & 73 & 96 & 1075 & 43 & 42 & 118 \\
\hline Male & 422 & 364 & 20 & 38 & 352 & 12 & 17 & 22 & 338 & 9 & 7 & 24 \\
\hline Female & 912 & 720 & 82 & 110 & 701 & 63 & 50 & 68 & 692 & 33 & 32 & 86 \\
\hline $\begin{array}{l}\text { Gender not } \\
\text { reported }\end{array}$ & 70 & 54 & 3 & 13 & 51 & 4 & 6 & 6 & 45 & 1 & 3 & 8 \\
\hline Left-handed & 141 & 119 & 9 & 13 & 120 & 9 & 5 & 6 & 112 & 3 & 4 & 11 \\
\hline Right-handed & 1192 & 962 & 92 & 138 & 927 & 66 & 63 & 87 & 913 & 38 & 34 & 101 \\
\hline Ambidextrous & 41 & 33 & 3 & 5 & 33 & 3 & 3 & 1 & 31 & 2 & 2 & 2 \\
\hline $\begin{array}{l}\text { Handedness } \\
\text { not reported }\end{array}$ & 30 & 24 & 1 & 5 & 24 & 1 & 2 & 2 & 19 & 0 & 2 & 4 \\
\hline $\begin{array}{l}\text { Age (mean } \pm \\
\text { SD) }\end{array}$ & $39 \pm 12.9$ & $\begin{array}{c}37 \\
\pm 12.8\end{array}$ & $\begin{array}{c}37 \\
\pm 12.5\end{array}$ & $\begin{array}{c}45 \\
\pm 12.7\end{array}$ & $\begin{array}{r}37 \\
\pm 12.7\end{array}$ & $\begin{array}{c}42 \\
+11.8\end{array}$ & $\begin{array}{c}41 \\
\pm 13.2\end{array}$ & $\begin{array}{r}42 \\
\pm 14.0\end{array}$ & $\begin{array}{c}37 \\
\pm 12.7\end{array}$ & $\begin{array}{r}42 \\
\pm 11.6\end{array}$ & $\begin{array}{c}43 \\
\pm 11.6\end{array}$ & $43 \pm 13.6$ \\
\hline
\end{tabular}

1 All numbers represent count data unless otherwise specified. 


\section{Figure 1}

Sample images used in the left/right judgement tasks

(A-D) Head-turning images at various orientations and in various postures. (E-F) Hand images depicting various postures. Photo credit: Juliet Gore; received from Noigroup archived images (www.noigroup.com)
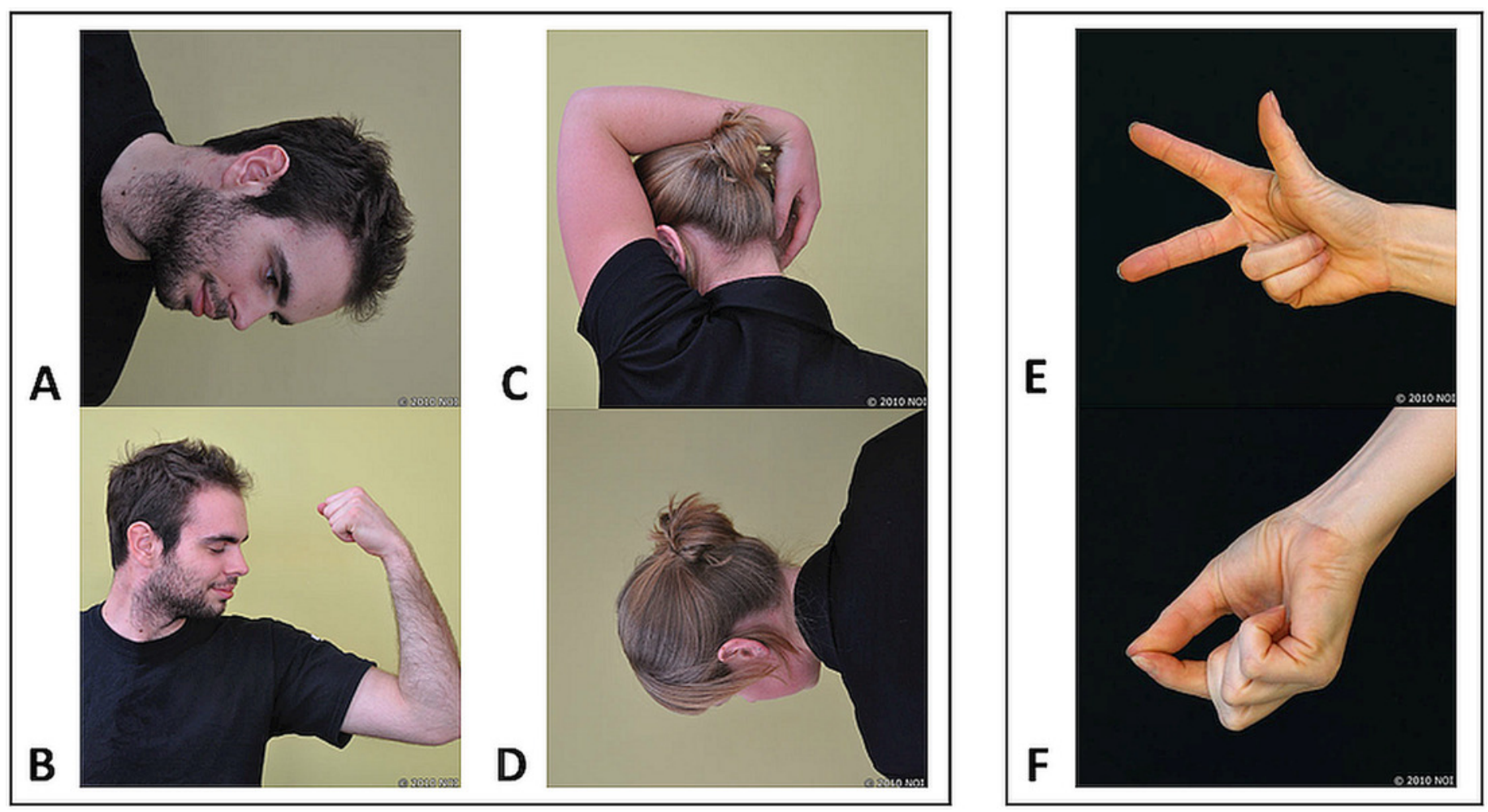
Figure 2

Effect of pain duration on left/right judgement performance

(A) Accuracy results for neck images. (B) Accuracy results for hand images. (C) Response time for neck images. (D) Response time for hand images. The red line indicates the significant post-hoc independent t-test findings for this comparison performed following an overall main effect of pain duration. Photo credit: Juliet Gore.
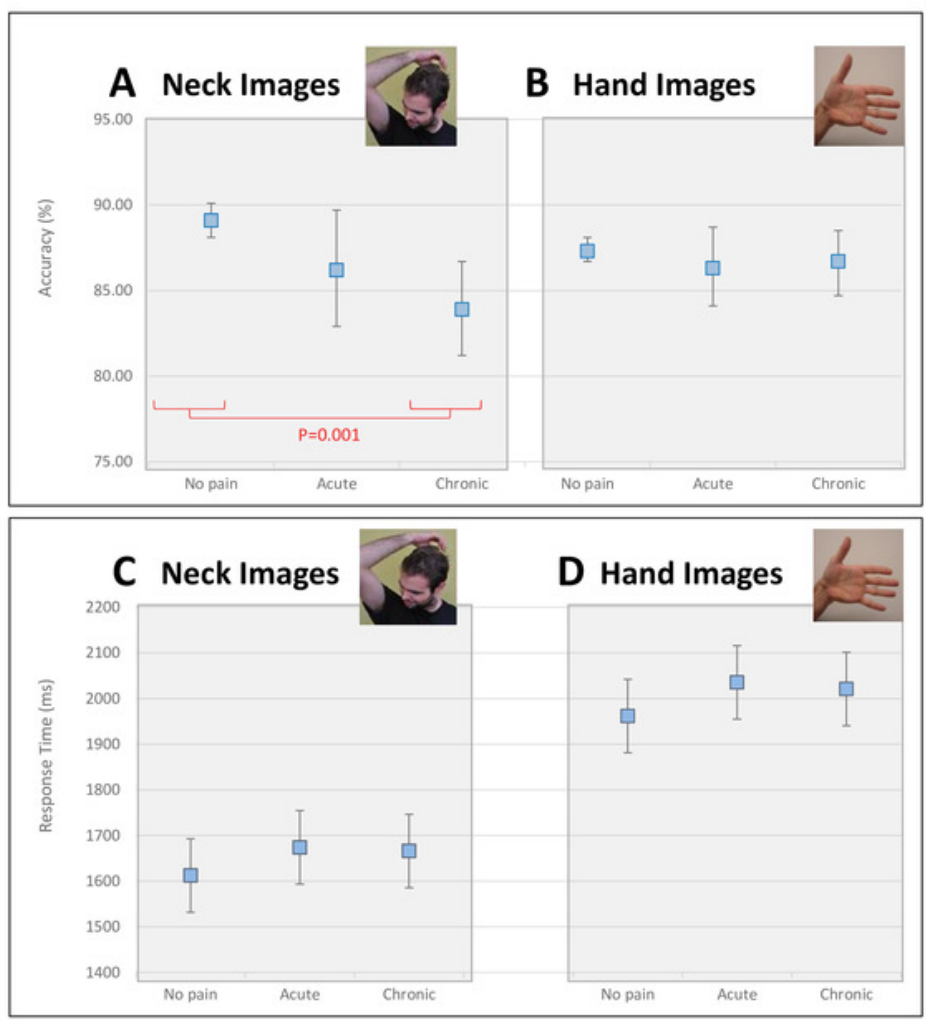
Figure 3

The effect of neck pain location (none, left-sided, right-sided, bilateral) on left/right neck judgement performance.

(A) Accuracy. (B) Response time. The blue line shows a main within subject effect of image type (direction of image head rotation). The red lines show significant posthoc independent ttest comparisons, following a main significant effect of pain location. Photo credit: Juliet Gore.

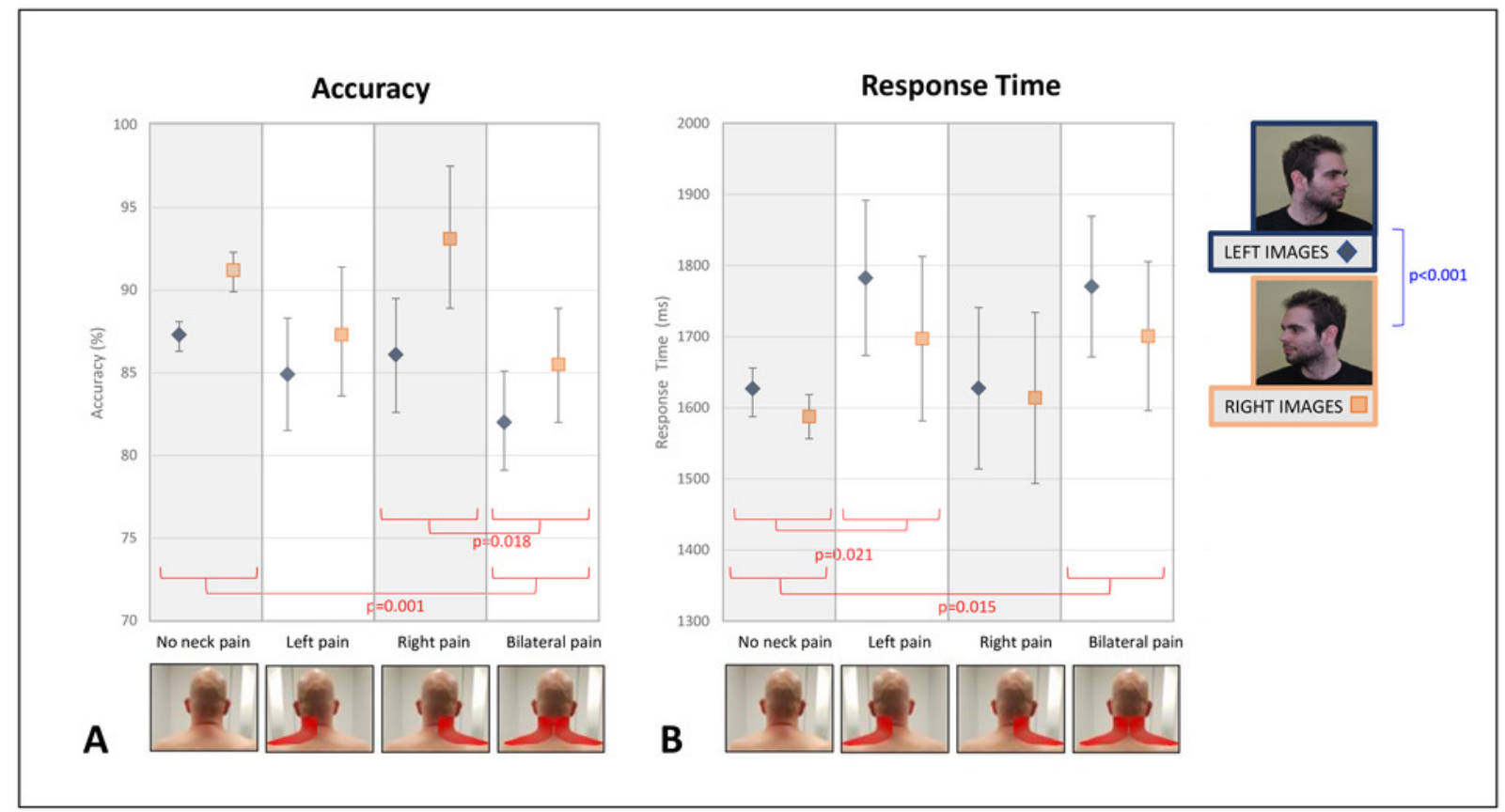


Figure 4

Effect of unilateral pain location (left- versus right-sided neck pain) on left/right neck judgement performance

(A) Accuracy. (B) Response time. The red lines indicate main between group effects of image type (left versus right head turning images). The dotted red line shows the posthoc independent t-test results showing significant performance differences between left- and right-sided pain only for right-sided neck images, following a significant interaction between image type and side of pain. Photo credit: Juliet Gore.

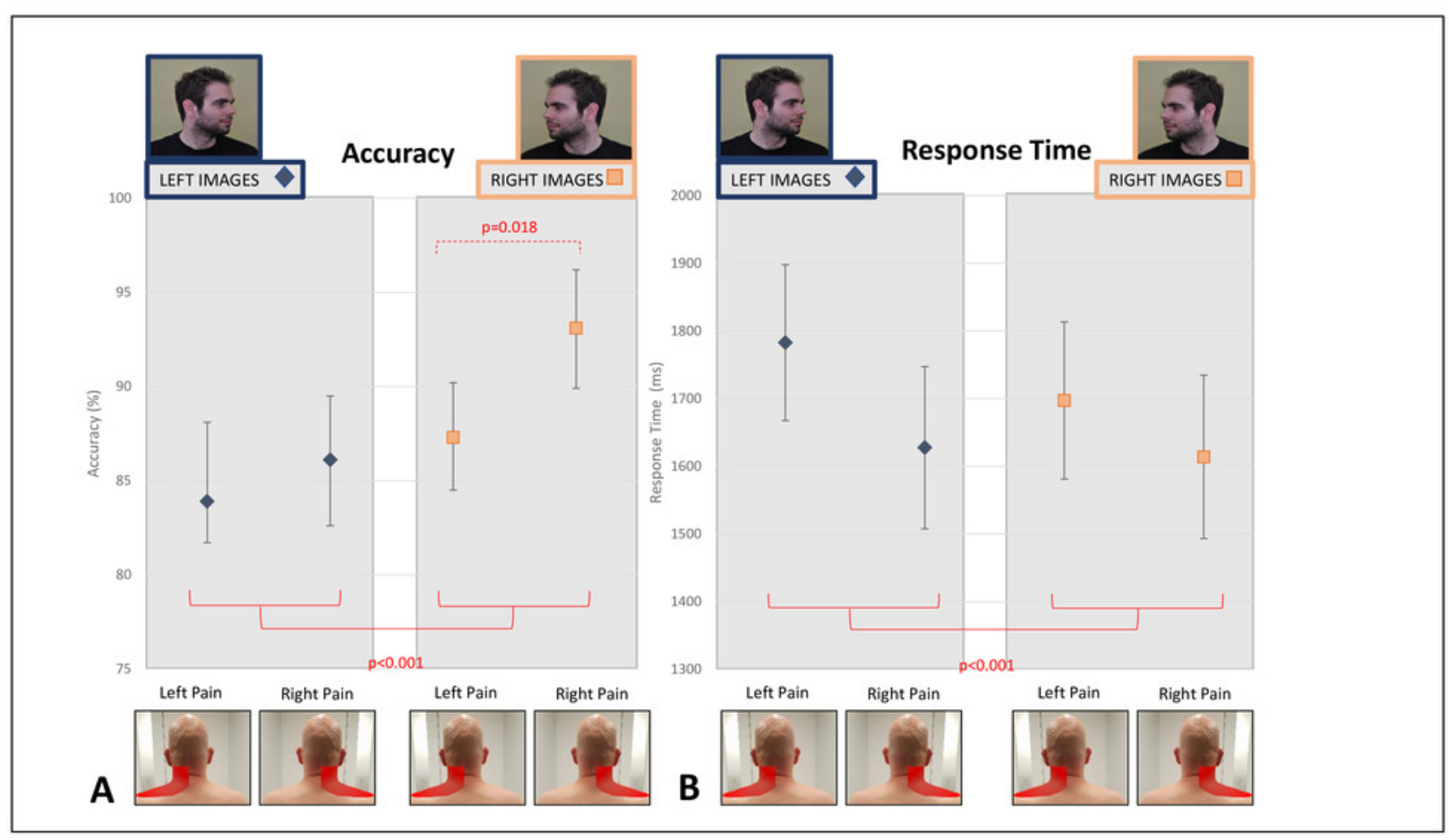

